\title{
Traumatic Tricuspid Regurgitation Treated by the Minimally Invasive Double Orifice Technique
}

\author{
Chan Kyu Lee, M.D. ${ }^{1}$, Jae Hoon Jang, M.D. ${ }^{2}$, Na Hyeon Lee, M.D. ${ }^{3}$, Seunghwan Song, M.D. ${ }^{3}$ \\ Departments of 'Trauma Surgery and '2Orthopedic Surgery, Pusan National University Hospital Trauma Center, Biomedical Research Institute, Pusan National \\ University School of Medicine; ${ }^{3}$ Department of Thoracic and Cardiovascular Surgery, Pusan National University Hospital, Biomedical Research Institute, Pusan \\ National University School of Medicine, Busan, Korea
}

\section{ARTICLE INFO}

Received January 23, 2020

Revised July 29, 2020

Accepted August 8, 2020

Corresponding author

Seunghwan Song

Tel 82-51-240-7267

Fax 82-51-243-9389

E-mail song77.sh@gmail.com

ORCID

https://orcid.org/0000-0002-7492-2097

\begin{abstract}
A 37-year-old man was transferred to our level I trauma center after a road traffic accident, presenting with right acetabular fracture, multiple rib fractures, epidural hemorrhage, and liver contusion. Severe traumatic tricuspid regurgitation was also discovered during the work-up for surgery. Our initial attempt at acetabular surgery failed when the patient experienced near cardiac arrest during anesthetic induction. It was hence decided that tricuspid valve repair should precede orthopedic surgery. Minimally invasive tricuspid valve repair using the double orifice technique was successfully performed. Subsequently, acetabular surgery was performed and he was discharged 35 days post-trauma without any complications.
\end{abstract}

Keywords: Blunt trauma, Tricuspid valve regurgitation, Tricuspid valve repair, Minimally invasive surgery, Double orifice technique

\section{Case report}

A 37-year-old man was transferred to the emergency department of our level I trauma center from a local hospital 20 hours after a traffic accident. Although the patient had lost consciousness at the time of the accident, his Glasgow Coma Scale score was 15 points (E4V5M6) at admission. Chest pain and tenderness were detected along the areas of the body where the seat belt was strapped. He experienced pain in and bruising on the right thigh and hip. Upon presenting to the emergency department, his vital signs were stable. There were no notable findings on an electrocardiogram or a chest X-ray exam.

Computed tomography (CT) performed at the other hospital and at Pusan National University Hospital Trauma Center showed mild epidural hemorrhage and a fracture of the occipital condyle. There were multiple fractures (with no significant dislocation) of the right first to fifth and left third to fifth ribs, mild bilateral hemothorax, and hematoma of the anterior mediastinum. Surgical treatment was required because of the fracture of the right acetabulum. He was identified as a patient with major trauma, with an injury severity score of 38 points.

Echocardiography was performed as cardiac contusion was suspected due to elevated troponin I levels $(0.078 \mu \mathrm{g} /$ L), as determined through the blood laboratory workup, and hematoma of the anterior mediastinum was detected on the CT scan. Echocardiography revealed papillary muscle rupture of the anterior cusp and severely eccentric tricuspid valve insufficiency, with patency of the foramen ovale (with a left-to-right shunt), right atrial enlargement, and tricuspid annular dilatation (Fig. 1). Left ventricular function was normal.

As the risk of surgery was confirmed to be low by the cardiology and anesthesiology teams, we decided to perform open reduction and internal fixation of the acetabular fracture with the patient in the left lateral decubitus position on the fifth day after admission because the patient was free of symptoms, and his vital signs were stable; moreover, cardiopulmonary bypass would have been difficult to perform due to the initial epidural hemorrhage. However, while changing the position of the patient to the lateral decubitus position in the operating room, the patient's mean blood pressure decreased to $<40 \mathrm{~mm} \mathrm{Hg}$ and 
his heart rate increased. Therefore, he was transferred to the intensive care unit, and after consultation with the attending cardiothoracic surgeon, we decided to perform orthopedic surgery after tricuspid valve repair. A brain CT examination revealed that the epidural hemorrhage was stable. On the 10th day after admission, cardiac surgery was performed for tricuspid regurgitation (TR). Minimally invasive cardiac surgery was initiated through a right mini-thoracotomy using a thoracoscopy. Cardiopulmonary bypass was established via the right femoral artery for the arterial line and the right femoral vein and right internal jugular vein for the venous lines. Heparin was injected at
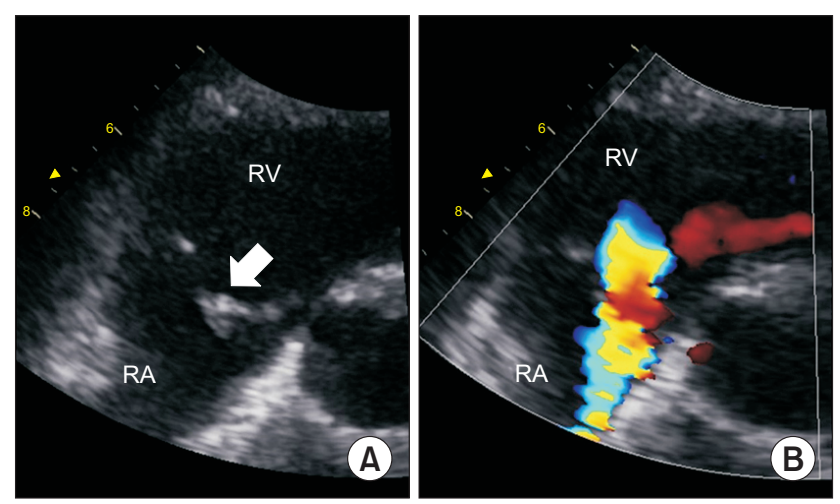

Fig. 1. (A, B) Zoomed-in image of the tricuspid valve in the parasternal short-axis view during preoperative echocardiography showing the ruptured papillary muscle (white arrow), flailing anterior leaflet, and severely eccentric tricuspid regurgitation. RA, right atrium; RV, right ventricle. only two-thirds of the usual dose to maintain an activated clotting time of just $>400$ seconds. After cardiac arrest was achieved by using an aortic cross-clamp with infusion of a cardioplegic solution, right atriotomy was performed for tricuspid valve evaluation. Rupture of the papillary muscle of the anterior leaflet of the tricuspid valve was observed, but the exact site of detachment was difficult to identify. We decided to repair the valve using the double orifice technique by placing a 4-0 polypropylene pledgeted suture from the edge of the anterior leaflet to the edge of the septal leaflet. At this point, a small degree of regurgitation was noted, as determined by an intraoperative saline test. As the annulus of the tricuspid valve was dilated, we selected a 32-mm Carpentier-Edwards MC3 ring (Edwards Lifesciences Corp., Irvine, CA, USA) to reduce the annular size. Thereafter, the competence of the tricuspid valve improved, and there was no regurgitation, as confirmed with an intraoperative saline test (Fig. 2) and intraoperative transesophageal echocardiography. The patient was weaned from cardiopulmonary bypass without difficulty. The cardiopulmonary bypass time was 156 minutes, and the aortic cross-clamping time was 87 minutes. Postoperative echocardiography did not reveal TR or stenosis (Fig. 3).

Five days after the resolution of TR, the patient underwent open reduction and internal fixation for the acetabular fracture in the left lateral decubitus position. The patient was discharged without any complications on post-trauma day 35, following a period of rehabilitation.

The patient provided written informed consent for publication of his clinical details and images.
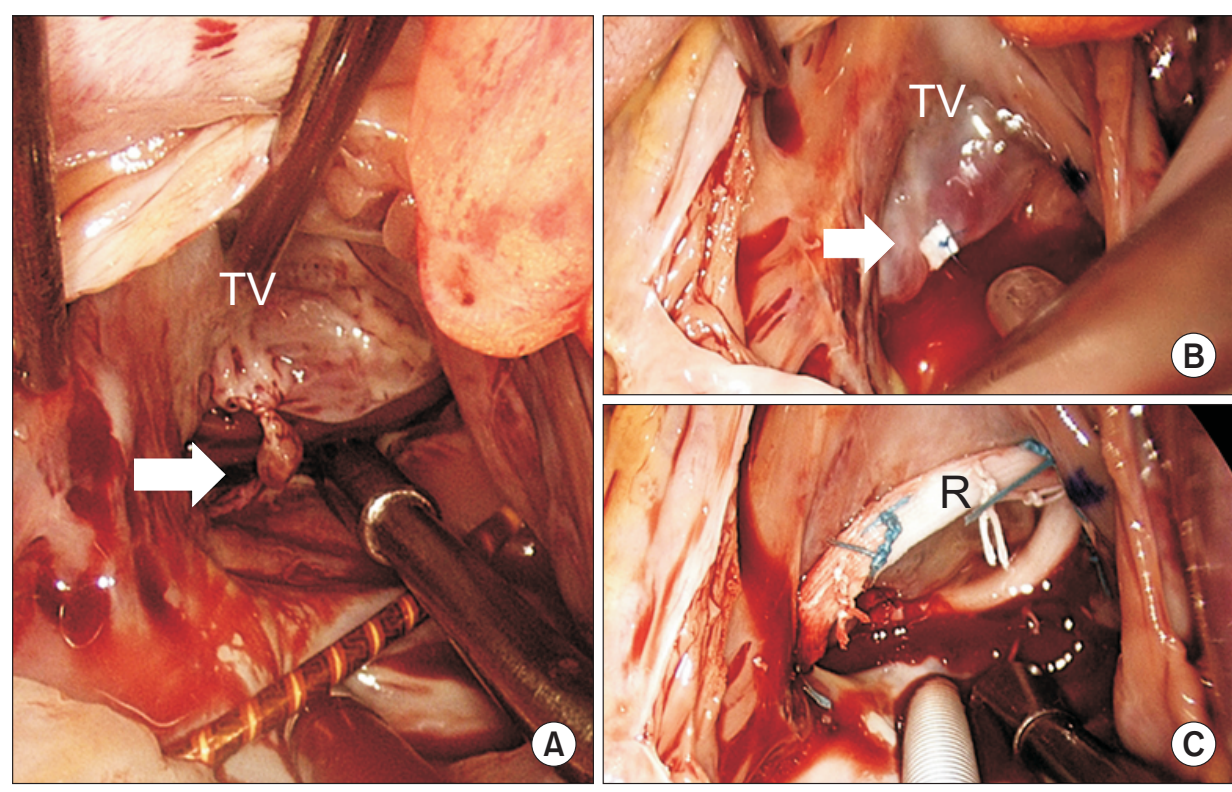

Fig. 2. Intraoperative thoracoscopy views. (A) Ruptured papillary muscle (white arrow) in the anterior leaflet of the tricuspid valve detected on right atriotomy. (B) Minimal regurgitation was detected in an intraoperative saline test after placing an edge-to-edge stitch with a 4-0 polypropylene pledgeted suture (white arrow). (C) Coaptation of the tricuspid valve was improved by reducing the diameter of the annulus using a 32-mm Carpentier Edward MC3 ring (Edwards Lifesciences Corp., Irvine, CA, USA). $T V$, tricuspid valve; $R$, artificial ring for annuloplasty. 

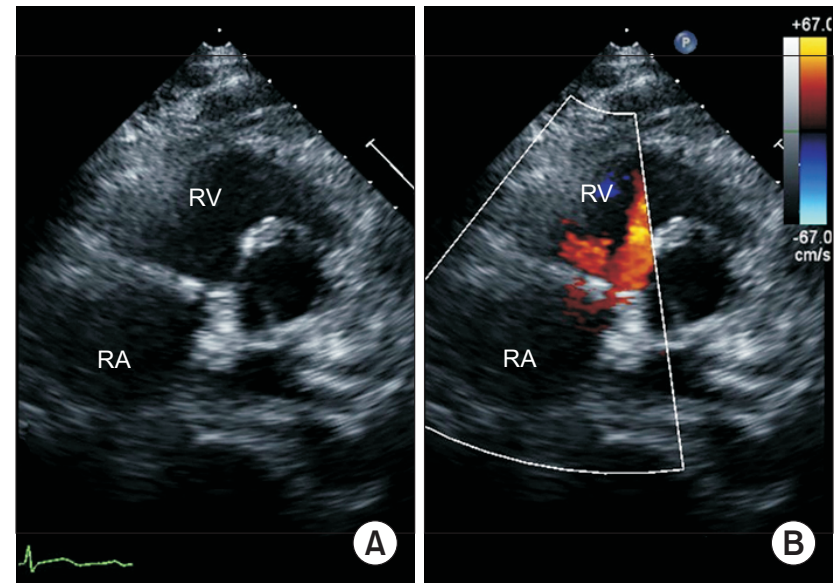

Fig. 3. (A, B) Parasternal short-axis view obtained during postoperative echocardiography shows good coaptation of the tricuspid valve without regurgitant flow. RA, right atrium; RV, right ventricle.

\section{Discussion}

Tricuspid insufficiency due to blunt chest trauma was first reported in 1829 by Williams [1], and approximately 150 cases have been reported worldwide [2]. The initial diagnosis of tricuspid insufficiency after cardiac trauma is challenging due to the possible absence of heart-related or other symptoms until several years later $[2,3]$. In our case, traumatic TR was diagnosed incidentally during an evaluation for cardiac contusion. The exact mechanism of TR due to blunt chest trauma is not known, but it is presumed that a sudden increase in the right atrial pressure accompanied by the heart being squeezed between the sternum and the vertebrae may play a vital role. This occurs most commonly during isometric systole when the heart is filled with blood and the valves are closed. The most common pathologic anatomical findings are rupture of the chordae tendineae (55\%), anterior papillary muscle injury (37\%), and tear of the leaflets (15\%). Enlargement of the annulus due to right ventricular dilatation is common and contributes to the worsening of regurgitation [3]. A recent study of trauma patients, especially those with other associated injuries, revealed that TR is increasingly being detected at an early stage with the use of more modern diagnostic modalities, such as echocardiography [4]. However, there is controversy regarding the precise indications for surgery, and how urgently such interventions should be initiated in the absence of symptoms.

In our case, although the vital signs were stable and there were no specific symptoms, TR was incidentally detected while performing echocardiography to rule out car- diac contusion. Due to the risk of bleeding from concurrent injuries, we decided to proceed with orthopedic surgery first. However, anesthetic induction and changing the patient's position led to a volume change in the right ventricle, resulting in near cardiac arrest in the operating room. Consequently, we decided to defer the orthopedic procedure to after cardiac surgery.

Although traumatic TR is rare, edge-to-edge repair, chordal transfer, annuloplasty, and replacement techniques have all been previously reported as TR repair techniques. However, with the exception of annuloplasty for functional $\mathrm{TR}$, the repair techniques for TR are not as varied as those for mitral valve repair $[3,5,6]$. Moainie et al. [4] introduced a double orifice technique using an Alfieri stitch and reported good results for traumatic TR. The double orifice technique was effective and technically simple for the treatment of TR caused by a flailing anterior or septal leaflet. However, with the Alfieri stitch alone, mild regurgitation could persist; therefore, annuloplasty may be necessary [4-6]. In our patient, repair was successful using the same technique.

Orthopedic surgery was planned after tricuspid valve repair, and the optimal time to perform surgery was within 2 weeks after the injury. Open reduction and internal fixation of the acetabular fractures required changing the position of the patient to the lateral position in the operating room. However, adopting a lateral position after conventional sternotomy is associated with risk of sternal instability. This complication was avoided by performing right mini-thoracotomy using thoracoscopy. This allowed clear visualization of the tricuspid valve and the right ventricle, obviating the need for the lateral position after conventional sternotomy. High success rates may be achieved with the minimally invasive approach via right lateral mini-thoracotomy due to excellent exposure and visualization of the tricuspid valve and subvalvular structures [7].

Acute cerebral hemorrhage is a relative contraindication for cardiac surgery. In view of the risk of cerebral hemorrhage during heparin infusion for cardiopulmonary bypass, we ensured that hemorrhage was stable prior to surgery through follow-up brain CT and administered a lower dose of heparin to prevent undue prolongation of the ACT. The unpredictable relationship between ACT and the heparin-dose response is a major concern. There is controversy regarding the heparin dose and ACT, especially with respect to trauma patients with excessive bleeding. In our case, two-thirds $(300 \mathrm{U} / \mathrm{kg})$ of the standard heparin dose $(400 \mathrm{U} / \mathrm{kg}$ ) was administered. The ACT was checked every 2-5 minutes and was maintained around 450 seconds 
during the surgery $[8]$.

In conclusion, most patients with traumatic TR are initially asymptomatic. However, owing to instability in the acute phase due to right ventricle failure, surgical treatment is often required. Therefore, if bleeding is stabilized in the acute phase, it is better to perform surgical treatment early. In our case, despite the hemorrhage in other organs in the acute phase, surgery could be performed without recurrence of bleeding. This was achieved by administering a lower dose of heparin than that commonly used in cardiac surgery after the absence of active bleeding was confirmed with CT. Using minimally invasive tricuspid valve repair with the double orifice technique as used in this patient, we were able to safely perform TR repair and orthopedic surgery sequentially, and our patient recovered well without any complications.

\section{Conflict of interest}

No potential conflict of interest relevant to this article was reported.

\section{Funding}

This work was supported by a clinical research grant from Pusan National University Hospital in 2019.

\section{ORCID}

Chan Kyu Lee: https://orcid.org/0000-0002-5765-1432

Jae Hoon Jang: https://orcid.org/0000-0002-2636-7957

Na Hyeon Lee: https://orcid.org/0000-0001-5242-1454

Seunghwan Song: https://orcid.org/0000-0002-7492-2097

\section{References}

1. Williams A. A case of post-traumatic tricuspid insufficiency. London Med Gaz 1829;4:78.

2. Ismailov RM, Weiss HB, Ness RB, Lawrence BA, Miller TR. Blunt cardiac injury associated with cardiac valve insufficiency: trauma links to chronic disease? Injury 2005;36:1022-8.

3. Alfieri O, de Bonis M, Lapenna E, Agricola E, Quarti A, Maisano F. The "clover technique" as a novel approach for correction of posttraumatic tricuspid regurgitation. J Thorac Cardiovasc Surg 2003; 126:75-9.

4. Moainie SL, Guy TS, Plappert T, Gorman JH 3rd, Gorman RC. Correction of traumatic tricuspid regurgitation using the double orifice technique. Ann Thorac Surg 2002;73:963-5.

5. Son KH, Son HS, Chung JH, Chung WJ, Sun K, Lee SH. Repair of posttraumatic tricuspid regurgitation using artificial chordae and an annuloplasty ring. Korean J Thorac Cardiovasc Surg 2008;41:48991.

6. Bang JH, Woo JS, Choi PJ, Park KJ, Jeong SS, Yi JH. Repair of ruptured papillary muscle of the tricuspid valve as a cause of tricuspid insufficiency following blunt chest trauma. Korean J Thorac Cardiovasc Surg 2010;43:413-6.

7. Minol JP, Boeken U, Weinreich T, et al. Isolated tricuspid valve surgery: a single institutional experience with the technique of minimally invasive surgery via right minithoracotomy. Thorac Cardiovasc Surg 2017;65:606-11.

8. Shore-Lesserson L, Finley A, Murphy GS, Gravlee GP. Anticoagulation for cardiopulmonary bypass. In: Gravlee GP, Davis RF, Hammon JW, Kussman BD, editors. Cardiopulmonary bypass and mechanical support: principles and practice. 4th ed. Philadelphia (PA): Wolters Kluwer; 2016. p. 463-96. 